\title{
A new method for characterizing hand dysfunction in cervical spondylotic myelopathy: a preliminary study
}

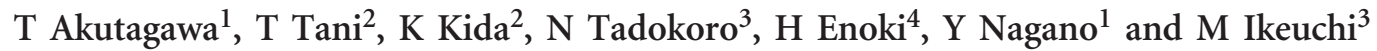

Study design: A case-control investigation.

Objectives: The objective of this study was to quantitatively study impaired ability to appropriately adjust pinch strength while holding a small object in patients with cervical spondylotic myelopathy (CSM).

Setting: Kochi Medical School Hospital, Japan.

Methods: The subjects consisted of 19 CSM patients who had frequent episodes of failing to grasp and hold small objects in their daily life (Group A), 13 CSM patients who did not experience such episodes (Group B) and 16 healthy subjects (Control Group). We continuously measured the dynamic internal pressure of a pneumatic rubber object called a blower pinched by the subject, following two different sets of instructions: (1) pinching with eyes open and with the minimal strength required to prevent dropping; and (2) maintaining a constant pinch strength at given levels with eyes closed.

Results: Compared with the other two groups, Group A subjects used a significantly $(P<0.01)$ greater pinch strength to avoid dropping the blower held with eyes open and showed a significantly $(P<0.01)$ greater deviation in pinch strength from the baseline values with eyes closed. These tendencies in Group A showed a significant correlation with the tactile perception threshold of the digits $(P<0.01)$ but not with impairment of rapid repetitive movements of the digits that reflects spasticity.

Conclusion: Our technique applied to CSM patients helps assess functional integrity primarily, if not exclusively, of the fasciculus cuneatus mediating the feedback signals from proprioceptive and cutaneous receptors in the digits, which are otherwise difficult to evaluate quantitatively.

Spinal Cord (2016) 54, 221-225; doi:10.1038/sc.2015.123; published online 28 July 2015

\section{INTRODUCTION}

Cervical spondylotic myelopathy (CSM) ranks first in terms of incidence of non-traumatic disorders of the spinal cord in adults. Most CSM patients lose fine finger movement, which appears to be common features of slowness and clumsiness seen in the majority of spastic patients. ${ }^{1-5}$ Although it occurs less frequently, CSM patients show the inability to control an appropriate pinch strength while holding an object, leading either to unintentional destruction of a fragile object with an exceedingly large pinch strength or to inadvertent dropping of an object because of insufficient pinch strength. This kind of disability could result, at least in part, from the involvement of the fasciculus cuneatus (FC), located laterally in the cervical dorsal columns causing the loss of proprioceptive and tactile information from the thumb and fingers. In fact, experiments using a dorsal column section model in monkeys revealed a decreased ability to precisely grasp objects between the thumb and the opposing forefinger. ${ }^{6-9}$ Despite its clinical importance, few studies ${ }^{10}$ have dealt with the objective assessment of this incapacity in CSM patients to date. We have therefore quantitatively studied the capacity to adjust pinch strength to have a better understanding of grasping failures in CSM patients. With a specially designed pressure recording device, we continuously measured the dynamic internal pressure of a pneumatic rubber object lifted and kept at the position by CSM patients and compared the results with healthy subjects. The technique should help elucidate the mechanism underlying this kind of disability.

\section{MATERIALS AND METHODS}

\section{Subjects}

We studied 57 hands from 32 CSM patients. The patients were classified into two subgroups, depending on whether they answered 'yes' or 'no' to the question 'Do you accidentally crush a soft, fragile object or drop a small object in your daily life?': (1) Group A answering 'yes' consisted of 31 hands from 19 patients (14 men) aged 50-79 years (mean, 67 years), and (2) Group B answering 'no' consisted of 26 hands from 13 patients ( 10 men) aged 53-80 years (mean, 66 years). Patients with other orthopedic problems (e.g., injury, deformation and amputation of fingers) and a history of peripheral or central nervous system disease were excluded. We also tested an age- and sex-matched Control Group consisting of 30 hands from 16 healthy volunteers (12 men) aged 59-77 years (mean, 67 years). All subjects agreed to participate in this study after reading and signing an informed consent form.

Functional assessments of the upper limb

(1) Motor function of the upper limbs was evaluated using the Japanese Orthopedic Association (JOA) scoring system for cervical myelopathy ${ }^{11}$ from 0 to 4 points: $0=$ unable to eat unaided; $1=$ able to eat using a spoon only; $2=$ able to eat using chopsticks with much difficulty; $3=$ able to eat using chopsticks with slight difficulty; and $4=$ normal. (2) The grip-and-release test ${ }^{3}$ evaluated the impairment of rapid repetitive finger movements simply by

${ }^{1}$ Department of Rehabilitation Center, Kochi Medical School Hospital, Kochi, Japan; ${ }^{2}$ Department of Orthopaedic Surgery, Kubokawa Hospital, Kochi, Japan; ${ }^{3}$ Department of Orthopaedic Surgery, Kochi Medical School, Kochi, Japan and ${ }^{4}$ Department of Physical Therapy, Faculty of Health and Welfare, Tokushima Bunri University, Tokushima, Japan Correspondence: T Akutagawa, Department of Rehabilitation Center, Kochi Medical School Hospital, Kohasu, Oko-cho, Nankoku City 783-8505, Kochi, Japan.

E-mail: aktgw81@gmail.com

Received 7 December 2014; revised 7 May 2015; accepted 15 June 2015; published online 28 July 2015 
testing how rapidly the patient can open and close the fists in $10 \mathrm{~s}$. (3) The Semmes-Weinstein monofilament testing assessed the threshold stimulus necessary for perception of light touch to deep pressure on the thumb, index and middle finger. (4) A maximum pinch strength was tested by pressing the tips of the thumb, index and middle finger on a pressure cuff.

\section{Imaging evaluation}

Spinal cord measurements at the level of maximal cord compression on T2-weighted magnetic resonance imaging (MRI) included the following: (1) anteroposterior (AP) diameter of the spinal cord on midsagittal images and (2) cord flattening ratio on axial images, defined as the minor axis length of the spinal cord divided by the major axis length.

\section{Continuous recording of pinch pressure}

We continuously measured the dynamic internal pressure of the object pinched by the subject. A specially designed pressure recording device consisted of a disk-shaped pneumatic rubber object called a blower (KA-16; HAKUBA Photo Industry Co., Ltd., Tokyo, Japan), a manometer, a pressure transducer (OHMEDA BD DTX plus; DATEX-OHMEDA PTY. LTD., Singapore) and an amplifier with a monitor for visual display (Addscope ATAC-210; Nihonkohden, Tokyo, Japan). The end of the blower has a projection through which weight can be hung with a thread (Figure 1). The pressure signals, sampled at $50 \mathrm{~Hz}$, were stored for subsequent off-line analyses.

\section{Experiment 1}

The subjects were seated on a chair in front of the experimental apparatus. They were then asked to lift the blower loaded with a $200 \mathrm{~g}$ weight above the table by means of a three-point pinch using the pulps of their thumb, index and middle finger. The investigator instructed them to keep the blower in the same position with the minimal pinch strength required to prevent slippage with eyes open.

\section{Experiment 2}

Under the same experimental settings as used in Experiment 1, subjects were instructed to lift the blower without the weight, keeping the blower in the same position with constant pinch strength of 10,20 and 30\% of maximum effort, which were chosen rather arbitrarily. With the help of the mercury level in the manometer, despite constant fluctuations, the subjects tried to adjust their voluntary muscle contractions to the respective target values. A few practice trials with eyes open usually enabled the subjects to maintain steady pinch strength for a certain level for at least $5 \mathrm{~s}$. In the subsequent test session, the procedure was performed with eyes open first, followed by with eyes closed. To help subjects time the closing of their eyes, the investigator gave a flash signal using a lighting system, which simultaneously injected a square-wave pulse into

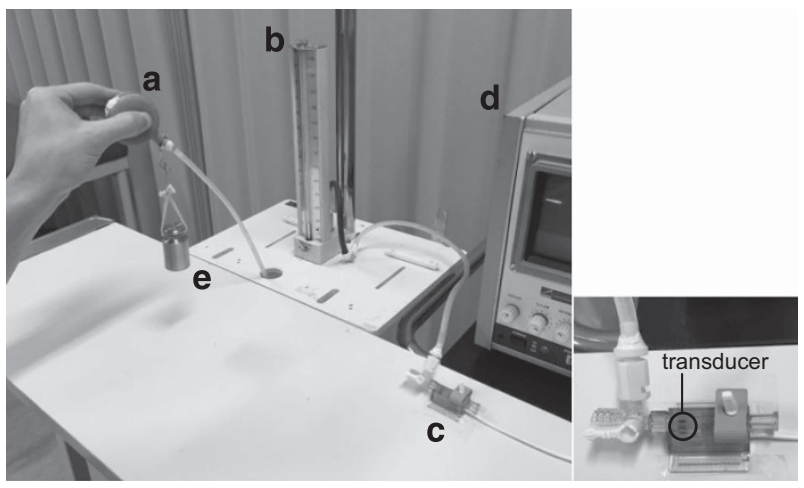

Figure $1 \mathrm{~A}$ general experimental design. A specially designed pressure recording system consists of a disk-shaped pneumatic rubber blower (a), a manometer (b), a pressure transducer (c) and an amplifier with a monitor for visual display (d). The end of the blower has a tiny projection through which weight (e) can be hung with a thread. the pressure recording device to indicate the beginning of the closed-eyes condition.

\section{Data analyses}

Using the digital data, we calculated the mean pinch pressure ' $P_{\mathrm{m} 1}$ ' for each subject in Experiment 1 over a 5-s period after the pinch pressure reached a fairly constant value with fluctuations of $<5 \mathrm{~mm} \mathrm{Hg}$. Then, we compared the $P_{\mathrm{m} 1}$ values between the three groups.

Similarly, in Experiment 2, we calculated the mean pinch pressure ' $P_{\mathrm{m} 2}$ ' for each subject over a 5-s period of the experiment immediately before the eyes were closed, which was taken as the baseline. We then calculated the root mean square (RMS) of the pinch pressure over a 5 -s period of the experiment immediately after the eyes were closed. As a measure of deviations in pinch pressure induced by changes from the open-eyes to closed-eyes condition, we defined the variability index ' $\mathrm{VI}$ ', which is given by $\mathrm{VI}=\mathrm{RMS} / P_{\mathrm{m} 2}$. The VIs for the procedures with 10,20 and $30 \%$ of the maximum effort are denoted by $\mathrm{VI}_{10 \%}, \mathrm{VI}_{20 \%}$ and $\mathrm{VI}_{30 \%}$, respectively.

All statistical analyses were performed using the $\mathrm{R}$ version 2.8.1 software ( $\mathrm{R}$ Foundation for Statistical Computing, Vienna, Austria). Significant differences in continuous data among the three groups (i.e., Group A, Group B and the Control Group) were identified using the multiple comparison tests by SteelDwass and between the two groups using Student's $t$-test or the Mann-Whitney $U$ test. Categorical data were compared using the Fisher's exact test. In addition, a multiple regression analysis was used to test whether the independent variables such as age, JOA functional assessment score, quickness in grip-andrelease test, tactile perception threshold of the digits and maximum pinch strength could be related to the experimental outcome variables that differed significantly among the three groups. The significance level was set at $P<0.05$.

\section{RESULTS}

\section{Functional assessments of the upper limb}

As shown in Table 1, the CSM patients in Group A had significantly lower values in the functional assessment score of the JOA (vs Group $\mathrm{B} ; P<0.01)$, quickness in the grip-and-release test $(P<0.01)$ and maximum pinch strength $(P<0.01)$ and significantly higher values in tactile perception threshold of the digits $(P<0.01)$, compared with those in the other two groups, although there was no significant difference in age $(P>0.05)$ among the three groups.

\section{Magnetic resonance imaging}

All CSM patients had unequivocal MRI evidence of cord compression. As shown in Table 2, maximal cord compressions in Group A were at $\mathrm{C} 3 / 4$ in five patients, at $\mathrm{C} 4 / 5$ in seven, at $\mathrm{C} 5 / 6$ in five and at $\mathrm{C} 6 / 7$ in two, whereas those in Group B were at $\mathrm{C} 2 / 3$ in one patient, $\mathrm{C} 3 / 4$ in

Table 1 Demographic characteristics and upper limb functions of study subjects in each group

\begin{tabular}{lccc}
\hline Variables & Group A & Group B & Control Group \\
\hline Sex (women/men) & $5 / 14$ & $3 / 10$ & $4 / 12$ \\
Age (years) & $67.0(6.8)$ & $65.5(8.4)$ & $66.9(5.4)$ \\
JOA functional assessment score & $1.7(0.9)^{\mathrm{a}}$ & $2.7(0.8)$ & $\mathrm{NA}$ \\
(points) & & & \\
Grip-and-release test (times) & $12.4(4.3)^{\mathrm{a}, \mathrm{b}}$ & $18.2(5.1)$ & $18.7(4.5)$ \\
Tactile sensation (Semmes- & $4.11(0.53)^{\mathrm{a}, \mathrm{b}}$ & $3.60(0.23)$ & $3.53(0.22)$ \\
Weinstein monofilament size) & & & \\
Maximum pinch strength & $160.5(41.2)^{\mathrm{a}, \mathrm{b}}$ & $202.1(39.9)$ & $213.9(34.5)$
\end{tabular}
( $\mathrm{mm} \mathrm{Hg}$ )

Values are mean (s.d.), except sex.

Abbreviation: JOA, Japanese Orthopedic Association; NA, not applicable.

aSignificant differences between Group A and Group B $(P<0.01)$.

bSignificant differences between Group $A$ and Control Group $(P<0.01)$. 
four, at C4/5 in two, at C5/6 in four and at C6/7 in two. The levels of maximal cord compression were not significantly different between Groups A and B $(P>0.05)$. Patients in Group A had significantly smaller anteroposterior diameter of the spinal cord compared with those in Group B $(3.9 \pm 1.5$ vs $5.1 \pm 1.1 \mathrm{~mm} ; P<0.05)$, although cord flattening ratios showed no significant difference between the two groups $(0.44 \pm 0.10$ vs $0.38 \pm 0.09 ; P>0.05)$.

\section{Experiment 1}

Subjects began increasing pinch force in response to the start command, and the pinch increased nearly monotonically up to a plateau within $5 \mathrm{~s}$, which continued changing slowly thereafter

Table 2 MRI measurement of cervical spinal cord

\begin{tabular}{lcc}
\hline Variables & Group A $(\mathrm{n}=19)$ & Group $B(\mathrm{n}=13)$ \\
\hline Intervertebral level of maximal cord compression & $($ no. of patients) & \\
C2/3 & 0 & 1 \\
C3/4 & 5 & 4 \\
C4/5 & 7 & 2 \\
C5/6 & 5 & 4 \\
C6/7 & 2 & 2 \\
& & \\
AP diameter (mean (s.d.)) (mm) & $3.9(1.5)^{\mathrm{a}}$ & $5.1(1.1)$ \\
Spinal cord flattening ratio (mean (s.d.)) & $0.44(0.10)$ & $0.38(0.09)$ \\
\hline
\end{tabular}

asignificant differences between Group A and B $(P<0.05)$.

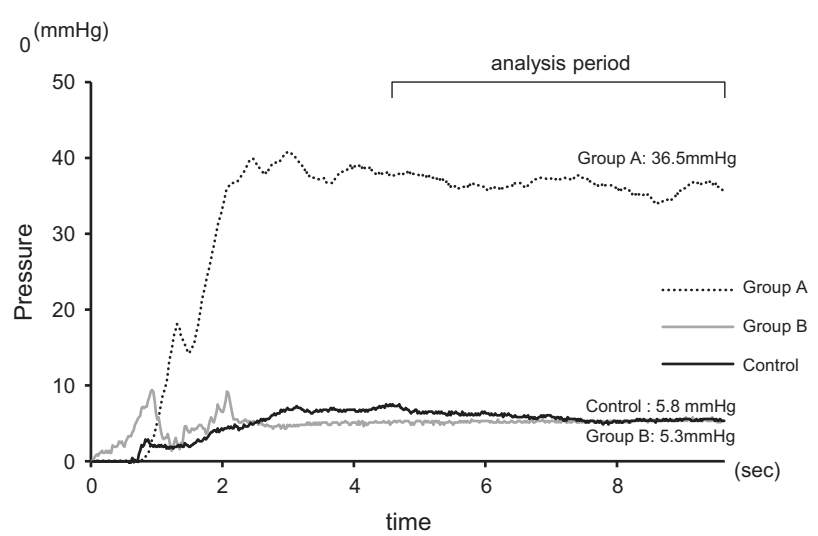

Figure 2 Sequential changes in the internal pressure of the blower recorded in three representative subjects, one from each of the three groups, during Experiment 1 . The mean pinch pressure, ' $P_{\mathrm{ml}}$ ' value, over a 5-s period (within parentheses) is shown for each subject.
(Figure 2). Over a 5-s period of the plateau, $P_{\mathrm{m} 1}$ averaged $22.43 \pm 10.80 \mathrm{~mm} \mathrm{Hg}$ in Group A, which was significantly larger compared with those in Group B $(12.65 \pm 6.01 \mathrm{~mm} \mathrm{Hg} ; P<0.01)$ and the Control Group $(9.95 \pm 4.73 \mathrm{~mm} \mathrm{Hg} ; P<0.01)$ (Table 3).

\section{Experiment 2}

As in Experiment 1, the pinch pressure tested with eyes open initially showed a rapid, progressive increase with some fluctuations and then reached a target value in all groups. With eyes closed, the subjects in Group A, unlike the other two groups, apparently failed to maintain steady pinch strength around a given level (Figure 3 ). There were no significant differences among the three groups about $\mathrm{VI}_{10 \%}$. Regarding $\mathrm{VI}_{20 \%}$ and $\mathrm{VI}_{30 \%}$, the values in Group $\mathrm{A}(8.80 \pm 6.26$ and $8.59 \pm 7.07 \%$ ) were significantly greater (all $P<0.01$ ) compared with the corresponding values in Group B $(4.36 \pm 2.69$ and $4.23 \pm 3.04 \%)$ and the Control Group $(3.05 \pm 1.98$ and $3.10 \pm 2.23 \%)$ (Table 3 ).

\section{Multiple regression analysis}

Table 4 summarizes the results of multiple regression analysis. Of the five independent variables, only the tactile perception threshold of the digits significantly correlated with ' $P_{\mathrm{m} 1}$ ' in Experiment 1 (regression coefficient, $0.51 ; P<0.01)$. In Experiment 2, the tactile perception threshold of the digits significantly correlated with $\mathrm{VI}_{20 \%}$ (regression coefficient, $0.38 ; P<0.05$ ) and $\mathrm{VI}_{30 \%}$ (regression coefficient, 0.52; $P<0.01$ ). Furthermore, the maximum pinch strength significantly correlated with $\mathrm{VI}_{20 \%}$ (regression coefficient, $-0.43 ; P<0.05$ ).

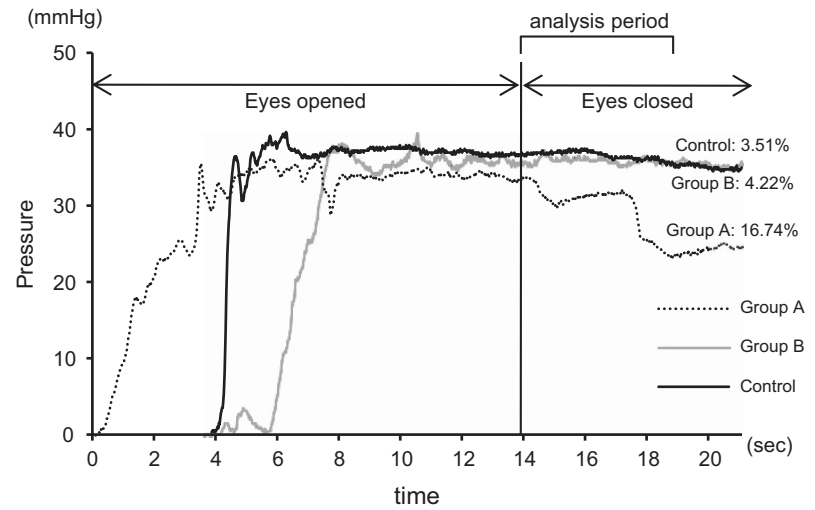

Figure 3 Sequential changes in the internal pressure of the blower recorded in three representative subjects, one from each of the three groups, during Experiment 2 with pinching at $20 \%$ of the maximal pinch strength. The variability index, ' $\mathrm{VI}_{20} \%$ ' value, over a 5 -s period (bracket) is shown for each subject.

Table 3 Ability to control pinch strength in each group

\begin{tabular}{lcc}
\hline Variables & Group A & Group B \\
\hline$P_{\mathrm{m} 1}$ in Experiment $1(\mathrm{~mm} \mathrm{Hg})$ & $22.43(10.80 ; 7.09-45.19)^{\mathrm{a}, \mathrm{b}}$ & $12.65(6.01 ; 5.63-27.57)$ \\
& & $9.95(4.73 ; 4.32-26.44)$ \\
$V I$ in Experiment 2 (\%) & $8.03(7.21 ; 1.59-34.21)$ & $6.50(4.37 ; 1.78-17.30)$ \\
$\mathrm{VI}_{10 \%}$ & $8.80(6.26 ; 1.78-23.07)^{\mathrm{a}, \mathrm{b}}$ & $4.36(2.69 ; 1.30-10.21)$ \\
$\mathrm{VI}_{20 \%}$ & $8.59(7.07 ; 1.28-35.54)^{\mathrm{a}, \mathrm{b}}$ & $4.23(3.04 ; 1.50-13.97)$ \\
$\mathrm{VI}_{30 \%}$ & $3.06(4.78 ; 1.79-27.78)$ \\
\hline
\end{tabular}

Values are mean (s.d.; range).

Abbreviations: $P_{m}$, mean pinch pressure; VI, variability index.

asignificant differences between Group $A$ and Control Group $(P<0.01)$.

bSignificant differences between Group A and B $(P<0.01)$. 
Table 4 Multiple regression analysis of the association between experimental outcome variables and independent variables

\begin{tabular}{|c|c|c|c|c|c|c|}
\hline \multirow{3}{*}{ Independent variables } & \multirow{2}{*}{\multicolumn{2}{|c|}{$\begin{array}{c}\text { Experiment } 1 \\
P_{m 1}\end{array}$}} & \multicolumn{4}{|c|}{ Experiment 2} \\
\hline & & & \multicolumn{2}{|c|}{$\mathrm{VI}_{20 \%}$} & \multicolumn{2}{|c|}{$\mathrm{VI}_{30 \%}$} \\
\hline & $\beta$ & P-value & $\beta$ & P-value & $\beta$ & P-value \\
\hline Age & 0.10 & NS & -0.08 & NS & 0.03 & NS \\
\hline JOA score & -0.01 & NS & 0.11 & NS & 0.06 & NS \\
\hline Grip-and-release test & -0.11 & NS & 0.11 & NS & 0.15 & NS \\
\hline Tactile sensation & 0.51 & $<0.01$ & 0.38 & $<0.05$ & 0.52 & $<0.01$ \\
\hline Maximum pinch force & 0.02 & NS & -0.43 & $<0.01$ & -0.20 & NS \\
\hline
\end{tabular}

Abbreviations: $\beta$, standardized partial regression coefficient; NS, not significant; VI, variability index.

\section{DISCUSSION}

Some CSM patients suffer from frequent episodes every day, in which they unintentionally destroy a fragile object by pinching it too firmly or dropping the object by pinching it too gently. This kind of disability in CSM patients, indicating the inability to accurately adjust the pinch strength to the weight of a hand-held object, has not received sufficient attention. To our knowledge, this is the first study to undertake an objective, quantitative assessment of this incapacity due to CSM. The presumed site of the lesion responsible for this functional change would be the FC, because previous experiments in monkeys demonstrated that a decreased ability to grasp objects precisely between the tip of the thumb and the opposing forefinger occurred as a result of the dorsal column section. ${ }^{6,12}$ Doita et al. ${ }^{10}$ reported that there was a significant correlation between the grade of proprioceptive impairment and the hand function scores in CSM patients, but they only qualitatively evaluated the proprioception in a conventional manner of testing how correctly the patient identified the various directions of the passive movement of a digit with their eyes closed. The present study attempts to delineate this functional incapacity by quantitatively examining to what extent the pinch strength is adjustable to given levels in CSM patients who have the symptoms under consideration (Group A), as compared with patients who do not have them (Group B) and healthy subjects (Control Group). Morphologically, patients in Group A had more severe spinal cord compression compared with those in Group B as shown by MRI evidence of significantly smaller anteroposterior diameter of the spinal cord at the maximal compression level.

The ' $P_{\mathrm{m} 1}$ ' values in Experiment 1 were significantly greater in Group A compared with that in the other two groups, indicating that the subjects in Group A tended to hold an object with unnecessarily high pinch strength to avoid dropping it. This tendency in Group A would account for the episodes in which they sometimes unintentionally destroy a fragile object while holding. Interestingly, Cooper et al. ${ }^{13}$ demonstrated with a key press experiment in monkeys that the forces exerted by the index finger against the keys increased, subsequent to the transection of the FC.

The functional importance of signals from afferents in controlling the pinch strength was also revealed in humans. Westling and Johansson, ${ }^{14}$ in a study analyzing how different surface properties of the object in terms of friction affect the pinch strength in healthy subjects, demonstrated that experimental digital nerve block raised the pinch strength. Also using digital anesthesia in healthy subjects, Augurelle et al. ${ }^{15}$ and Monzée et al. ${ }^{16}$ studied the contributions of cutaneous feedback in grip force control. They demonstrated, either with the apparatus containing an accelerometer and strain gage ${ }^{15}$ or with a linear motor equipped with load cells, ${ }^{16}$ that cutaneous afferents have an essential role in adapting grip and lifting forces to surface friction and object weight. Furthermore, Witney et al., ${ }^{17}$ in their review of literature, concluded that feedback from cutaneous afferents is crucial for successful feedforward control of hand-arm coordination.

In Experiment 2, we tested how steadily the subjects could maintain their pinch strength around set values when deprived of visual cues. As shown by significantly greater values of ' $\mathrm{VI}_{20 \%}$ ' and ' $\mathrm{VI}_{30 \%}$ ' in Group A compared with that in the other two groups, which indicates greater deviations in pinch strength from the baseline, subjects in this group tended to rely on their vision more than the other two groups for controlling the muscles of the hand. This finding implies that patients in this group have limited information available from proprioceptive and cutaneous receptors in the digits. Similarly, in one study on a patient with multiple sclerosis, Hashimoto et al. ${ }^{18}$ demonstrated that the involvement of the dorsal columns considerably affected the ability of subjects to keep the pinch strength steady while holding an object with their eyes closed.

As expected, our multiple regression analysis showed that the higher the tactile perception threshold of the digits, the greater the ' $P_{\mathrm{m} 1}$ ' value tended to be in Experiment 1 and ' $\mathrm{VI}_{20 \%}$ ' and ' $\mathrm{VI}_{30 \%}$ ' in Experiment 2. These results indicate that a continuous flow of information from cutaneous as well as proprioceptive afferents is necessary for proper motor control. The multiple regression analysis also showed a significant negative correlation between the maximum pinch strength and the ' $\mathrm{VI}_{20 \%}$ ' value, suggesting that as the weakness of the hand muscles becomes more prominent, the accuracy of muscle control decreases. In fact, the pyramidal tract section in monkeys showed similar effects on grip force control to those following the dorsal column section. ${ }^{19}$

In contrast, neither the JOA score nor the grip-and-release test, both of which mainly reflect limb spasticity, correlated significantly with ' $P_{\mathrm{ml}}$ ' in Experiment 1 and 'VIs' in Experiment 2. Unlike spasticity, dysfunctions of the proprioceptive and tactile feedback systems impair the ability to maintain or modulate position or force, ${ }^{20}$ because the systems generally operate relatively slowly.

Therefore, the present data on CSM patients confirm the clinical importance of our technique as a measure of functional integrity, mainly, if not exclusively, of the FC transmitting proprioceptive and tactile feedback signals, which are otherwise difficult to evaluate quantitatively. As an added inference, the technique may also provide a useful measure in feedback rehabilitation for this kind of disability, although further validation studies are needed before this method can be applied in clinical practice.

\section{Study limitations}

First, we divided the CSM patients into two groups according to one 'yes' or 'no' answer to one simple question. More detailed information about patients' disabilities in their daily lives to discriminate Group A from Group B would be desirable. Second, we quantitatively measured only touch sensation not proprioception. The device developed and well validated by Wycherley et al., ${ }^{21}$ if available, would be useful in measuring finger proprioception. Third, repeated studies were not conducted to analyze test-retest reproducibility. Subjects underwent a series of tests, conducted only once, after a few practice trials. Fourth, a specially designed pressure recording device used in the present study is not commercially available at the moment, which may partially preclude other investigators from reproducing our technique.

This study nonetheless contributes to quantitative assessment of a clinically important disability that currently receives little attention in CSM patients and, thereby, will at least provide some basis for further 
refinement in its practical application to CSM evaluation and rehabilitation.

\section{CONCLUSIONS}

Continuously measuring the internal pressure of a pneumatic rubber object held stationary against gravity, CSM patients with grasping failures in daily life applied significantly greater pinch strength to prevent accidental slips and maintained the required pinch strength less constantly compared with patients without such disabilities and healthy subjects. The technique needs further validation studies before it can be applied in feedback rehabilitation for this kind of disability.

\section{DATA ARCHIVING}

There were no data to deposit.

\section{CONFLICT OF INTEREST}

The authors declare no conflict of interest.

1 Crandall PH, Batzdorf U. Cervical spondylotic myelopathy. J Neurosurg 1966; 25 57-66.

2 Sahrmann SA, Norton BJ. The relationship of voluntary movement to spasticity in the upper motor neuron syndromes. Ann Neurol 1977; 2: 460-465.

3 Ono K, Ebara S, Fuji T, Yonenobu K, Fujiwara K, Yamashita K. Myelopathy hand: new clinical signs of cervical cord damage. J Bone Joint Surg 1987; 69-B: 215-219.

4 Tani T, Ishida K, Ushida T, Yamamoto H. Intraoperative electroneurography in the assessment of the level of operation for cervical spondylotic myelopathy in elderly. $J$ Bone Joint Surg (Br) 2000; 82: 269-274.

5 Tani T, Ushida T, Taniguchi S, Kimura J. Age related shift in the primary sites of involvement in cervical spondylotic myelopathy from lower to upper levels. J Neurol Neurosurg Psychiatry 2002; 73: 316-318.
6 Ferraro A, Berrera SE. Effects of experimental lesions of the posterior columns in rhesus monkeys. Brain 1934; 57: 307-332.

7 Dubrovsky B, Davelaar E, Garcia-Rill E. The role of dorsal columns in serial order acts. Exp Neurol 1971; 33: 93-102.

8 Vierck CJ Jr. Interpretations of the sensory and motor consequences of dorsal column lesions. In: Gordon G (ed). Active Touch. Pergamon Press: Oxford, UK, 1978 , pp 139-159.

9 Devidoff RA. The dorsal columns. Neurology 1989; 39: 1377-1385.

10 Doita M, Sakai H, Harada T, Nishida K, Miyamoto H, Kaneko T et al. The influence of proprioceptive impairment on hand function in patients with cervical myelopathy. Spine 2006; 31: 1580-1584.

11 Japanese Orthopaedic Association. Scoring system for cervical myelopathy. Nippon Seikeigeka Gakkai Zasshi 1994; 68: 490-503.

12 Vierck CJ Jr. Comparison of forelimb and hindlimb motor deficits following dorsa column section in monkeys. Brain Res 1978; 146: 279-294.

13 Cooper BY, Glendinning DS, Vierck CJ Jr. Finger movement deficits in the stumptail macaque following lesion of the fasciculus cuneatus. Somatosens Mot Res 1993; 10: $17-29$.

14 Westling G, Johansson RS. Factors influencing the force control during precision grip. Exp Brain Res 1984; 53: 277-284.

15 Augurelle A-S, Smith AM, Lejeune T, Thonnard J-L. Importance of cutaneous feedback in maintaining a secure grip during manipulation of hand-held objects. J Neurophysiol 2003; 89: 665-671.

16 Monzée J, Lamarre Y, Smith AM. The effects of digital anesthesia on force control using a precision grip. J Neurophysiol 2003; 89: 672-683.

17 Witney AG, Wing A, Thonnard J-L, Smith AM. The cutaneous contribution to adaptive precision grip. Trends Neurosci 2004; 27: 637-643.

18 Hashimoto R, Kanho M, Fujimoto K, Tanaka Y. Left hand clumsiness due to disturbance of kinesthesia after damage to the dorsal column of the high cervical cord. Rinsho Shinkeigaku 1997; 37: 319-325.

19 Mountcatsle VB. Central nervous system mechanisms in mechanoreceptive sensibility. In: Brookhart JM, Mountcatsle VB (eds). Handbook of Physiology, vol. 3. Sensory processes, part 2. American Physiological Society: Bethesda, MD, USA, 1984, pp 789-878.

20 Ghez C. The control of movement. In: Kandel ER, Schwartz JH, Jessell TM (eds), Principles of Neural Science, 3rd edn. Elsevier: Tokyo, Japan, 1991, pp 533-547.

21 Wycherley AS, Helliwell PS, Bird HA. A novel device for the measurement of proprioception in the hand. Rheumatol 2005; 44: 638-641. 